\title{
Context-Based Fall Detection using Inertial and Location Sensors
}

\author{
Hristijan Gjoreski, Mitja Luštrek, Matjaž Gams \\ Department of Intelligent Systems, Jožef Stefan Institute \\ Jamova cesta 39, 1000 Ljubljana, Slovenia \\ \{hristijan.gjoreski, mitja.lustrek, matjaz.gams\}@ijs.si
}

\begin{abstract}
Falls are some of the most common sources of injury among the elderly. A fall is particularly critical when the elderly person is injured and cannot call for help. This problem is addressed by many fall-detection systems, but they often focus on isolated falls under restricted conditions, neglecting complex, real-life situations. In this paper a combination of body-worn inertial and location sensors for fall detection is studied. A novel context-based method that exploits the information from both types of sensors is designed. The evaluation is performed on a real-life scenario, including fast falls, slow falls and fall-like situations that are difficult to distinguish from falls. All the possible combinations of six inertial and four location sensors are tested. The results show that: (i) context-based reasoning significantly improves the performance; (ii) a combination of two types of sensors in a single physical sensor enclosure seems to be the best practical solution.
\end{abstract}

Keywords: Context-based reasoning, Fall detection, Inertial sensors, Location sensors, Activity recognition.

\section{$1 \quad$ Introduction}

Falls are some of the most critical health-related problems for the elderly [3]. Approximately $28-35 \%$ of people over the age of 65 fall each year, and this proportion increases to $32-42 \%$ in those aged more than 70 years [20]. About $20 \%$ of all the fall accidents that involve an elderly person require medical attention [6]. Furthermore, falls and the fear of falling are important reasons for nursing-home admission [18]. Falls are particularly critical when the elderly person is injured and cannot call for help. These reasons, combined with the increasing accessibility and miniaturization of sensors and microprocessors, is driving the development of fall-detection systems.

Even though fall detection (FD) has received significant attention in recent years, it still represents a challenging task for two reasons. First, there are several everyday fall-like activities that are hard to distinguish from fast falls. Most of the current approaches define a fall as having greater accelerations than normal daily activities. However, focusing only on a fast acceleration can result in many false alarms during fall-like activities with fast acceleration, such as sitting down quickly or lying down 
on a bed quickly. The second reason why FD is challenging is that not all falls are characterized by a fast acceleration. Rubenstein et al. [16] showed that $22 \%$ of the falls experienced by the elderly are slow and are caused by dizziness and vertigo (13\%), and drop attacks (9\%). Therefore, the detection of slow falls should be an intrinsic part when creating a successful fall-detection system.

To overcome the problems of the existing fall-detection methods discussed above, we propose a new approach to FD by combining body-worn inertial and location sensors, named CoFDILS (Context-based Fall Detection using Inertial and Location Sensors). Our approach uses the context information from the both types of sensors to determine whether a fall has occurred. It exploits body accelerations, location and atomic activities to detect a fall. The evaluation was performed on a special real-life scenario that includes fast falls, slow falls and non-fall situations that are difficult to distinguish from falls. In addition, we tested 1023 possible body-placement combinations of six inertial and four location sensors in order to find the best-performing sensor placements for FD and therefore to achieve the lowest sensor burden on the user. The results showed that by combining the two types of sensors it is possible to detect complex fall situations and that the context-based reasoning significantly improves the performance.

The paper is organized as follows. Firstly, an overview of the related studies for FD is presented in Section 2. In the next two sections, the sensor equipment (Section 3 ) and the architecture of our system (Section 4) are described. Next, the preprocessing of the raw data is presented in Section 5. In the next two sections we describe the context components (Section 6) and the methodology (Section 7). After that, the experimental setup, the results and discussions are presented in Section 8 and 9. Finally, we conclude this work and give directions for future work in Section 10.

\section{$2 \quad$ Related Work}

FD approaches can be divided into those using non-wearable and wearable (i.e., bodyworn) sensors. The most common non-wearable approach is camera-based $[10,15]$. Although this approach is physically less intrusive to the user compared to the bodyworn sensors, it suffers from issues such as low image resolution, target occlusion and time-consuming processing. However, often the biggest issue is user privacy: the user has to accept the fact that a camera will record him/her.

Most of the studies for FD are based just on inertial sensors. Usually, they are focused only on fast falls $[13,23]$, which are not difficult to detect using the acceleration signal. The non-fall events used to test for false positives are usually normal, everyday activities $[8,15]$, not events chosen specifically because they are easily mistaken for falls. In contrast, we used complex falls and safe events that appear like falls. An example where FD was evaluated on events difficult to recognize as falls or non-falls is the work by Li et al. [11]. By applying thresholds to two inertial sensors, they detected a fall with an accuracy of $90.1 \%$. The recall value of their method on a fall event ending with sitting is $50 \%$ and for a non-fall event, quickly lying on a bed, 
is $40 \%$. By combining one inertial and one location sensor, we were able to achieve $99 \%$ and $100 \%$, on similar events, respectively.

A combination of inertial and location sensors was described in Zinnen et al. [23]. However, their goal was activity recognition for car-quality control and they did not deal with FD. Their approach was based on high-level primitives that were derived from a reconstructed human-body model by using inertial sensor data. The location data was mainly used to estimate the person's location near the car.

We are not aware of any prior publication that studies a combination of body-worn inertial and location sensors for FD, except ours [14]. There, we focused on locationbased FD, and we considered only a single accelerometer to detect the impact of the fall and the orientation of the user. The main advantages of the study presented here compared to our previous work are: (i) a machine-learning model that recognizes the activity of the user; (ii) a thorough analysis of the system's complexity and invasiveness to the user by analyzing the performance of all the possible body-placement combinations of 10 sensors; and (iii) an explicit presentation of the context-based reasoning algorithm, the core of our system.

A context-based approach to FD is presented in the study by Li et al. [12]. However, they used a different fall-detection method and different types of sensors to extract the context information, compared to our approach. In particular, they used 5 bodyworn accelerometers and 2 environmental sensors that monitor the vibration of the furniture. They combined the user's posture information, extracted from the accelerometers, and the context information, extracted from the environmental sensors, in order to detect the fall situations. Although they also analyzed slow falls and fall-like situations, their evaluation was performed on only 3 test subjects; while we tested our method on 11 subjects. The advantage of our location system, compared to the environmental sensors, is that it provides richer information about the user's situation, e.g., the user's location, the sensor's height, etc. The environmental sensors used in their research can only inform about the presence/absence of the user at a specific location where the sensor is installed. We tested all the combinations of 10 sensors and found a satisfactory performance with single sensor enclosure, while they analyzed only the fixed 5 accelerometer placements on the body.

To summarize, the improvements of our FD approach upon most related work are the following:

- combining two types of body-worn sensors: inertial and location,

- context reasoning about the user's situation,

- analysis of the FD performance for all combinations of 10 sensor body placements,

- machine learning activity-recognition model as a part of the FD,

- evaluation on a complex test scenario, including events such as slow falls and falllike events that may be difficult to distinguish from falls.

\section{Sensor Equipment}

The CoFDILS sensor equipment consists of inertial and location sensors (Fig. 1). The two types of sensors were chosen because inertial sensors are relatively cheap and 
portable, and the location sensors provide rich information about the user, without significantly compromising the user's privacy (like with the cameras).

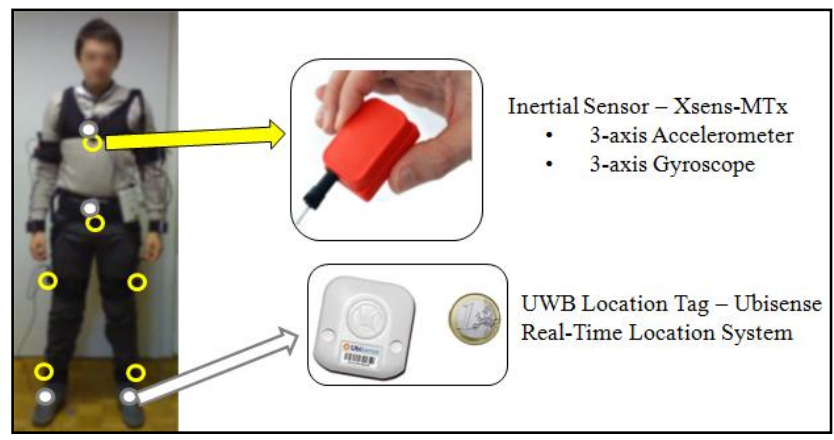

Fig. 1. Sensor equipment. The empty yellow circles represent the inertial sensors and the filled white circles represent the location tags.

Six inertial sensors were placed on the chest, waist, left thigh, right thigh, left ankle, and right ankle (non-filled circles in Fig. 1). Since only activities that are associated with the user's legs and torso were studied, the arm- and wrist-sensor placements were not considered. The inertial sensor equipment consisted of body-worn Xsens-MTx sensors [22], but the methods developed for this research are general and can be applied to any type of inertial sensor.

Four location tags were placed on the chest, waist, left ankle and right ankle (filled circles in Fig. 1). They emit UWB radio signals, which are detected by sensors fixed in the corners of a room. The tags are detected by the location system and their coordinates are computed. The location system used in CoFDILS is Ubisense [19]; it is a real-time location system used to track subjects indoors. Note that for simplicity the term sensor is also used for the body-worn location tag.

The data-sampling frequency of the sensors was set to $10 \mathrm{~Hz}$ because of Ubisense's hardware limitations. Although the inertial sensors do not have the same limitation, the data is sampled at the same frequency to simplify the synchronization.

\section{System Architecture}

The architecture of CoFDILS is shown in Fig. 2. First, the data from both types of sensors is stored and preprocessed. Next, the flow of data splits into two. On the top, firstly, a feature extraction is performed and the constructed feature vector is fed to the activity-recognition (AR) classification model, which recognizes the activity of the user. At the bottom, context-based reasoning about the user's situation is performed. The context reasoning analyzes the activity of the user and additional context information from the preprocessed data. The motivation is that the context information depends on the type of sensors. Inertial sensors provide body-movement information and the detection of a rapid-acceleration fall pattern, i.e., a threshold-based approach (TBA). Location sensors provide the location of the user in the room. The 
system evaluates the information from various sources in light of its contexts and concludes whether a fall alarm should be issued. Each module in Fig. 2 is presented in more detail in the sections that follow.

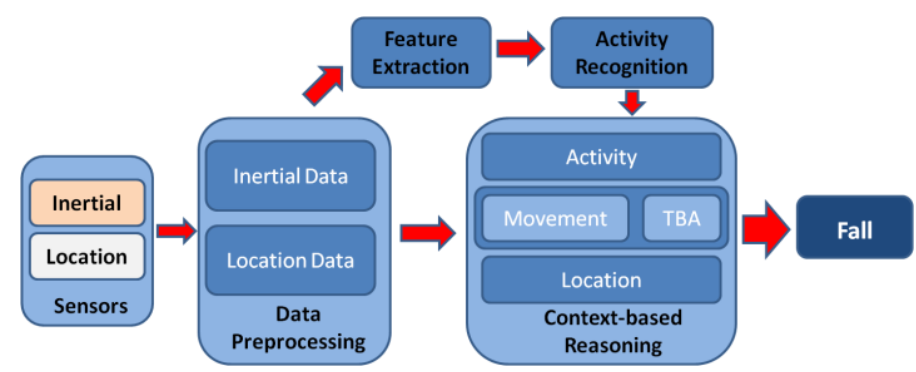

Fig. 2. CoFDILS architecture. TBA - Threshold-based approach.

\section{$5 \quad$ Data Preprocessing}

\subsection{Inertial Data}

An inertial sensor provides the raw data that consists of 3-axis accelerometer data and 3 -axis gyroscope data. It measures the accelerations and the angular velocities represented in three directions.

The raw data was filtered with low-pass and high-pass filters. The low-pass filter removes the movement of the sensors, which leaves only the gravity component. This information is useful, especially for an assessment of the sensor-inclination angles. In contrast, the high-pass filter removes the gravity and only the sensor movements are left. These filters were applied separately: if the gravity component is needed, the low-pass filtered data is used; otherwise, the high-pass filtered data is used.

Finally, an overlapping sliding-window technique was applied for the AR. This means that a one-second window moves across the stream of data, advancing by half its length for each step.

\subsection{Location Data}

The Ubisense's output consists of the 3D coordinates of the sensors that are attached to the user's body. In a typical open-environment, the localization accuracy is about $15 \mathrm{~cm}$, but in practice it may occasionally drop to $200 \mathrm{~cm}$ or more. Therefore, filtering was performed in order to tackle the problems with the Ubisense system [9].

First, a median filter computed each coordinate as the median of the measured values in a time window. This type of filtering removes large, short-term deviations of a measured coordinate from the true one. Second, the coordinates were corrected with a filter enforcing anatomic constraints based on the user's height and the body proportions. After that, a Kalman filter was used to smooth the data and correct some of the errors. Finally, the same overlapping sliding-window technique was applied. 
The most important novelty in our fall-detection method (CoFDILS) is based on the use of the context information. In general, a context is defined as any information that can be used to characterize the circumstances in which an event occurs [2]. In CoFDILS, the context information consists of three components: (i) the user's body accelerations, (ii) the user's activities and (iii) the location of the user.

\subsection{Body Accelerations}

\section{Threshold-based Approach}

The threshold-based approach (TBA) is used as one of the components in CoFDILS, as well as a baseline for comparison. The rationale for this method is that the acceleration pattern during a typical fall (i.e., fast, uncontrolled) is a decrease in the acceleration (free fall) followed by a rapid increase (impact with the ground). For our implementation of the TBA, the difference between the maximum and minimum accelerations within a one-second window was calculated. If the difference exceeded the threshold and the maximum appeared after the minimum, a fall was declared. The threshold was chosen empirically based on preliminary data [4].

\section{Body Movement}

During motion the accelerometers produce a changing acceleration signal and the fiercer the motion, the greater the change in the signal. Using these changes a feature is extracted: Acceleration Vector Changes (AVC) [4]. This feature sums up the differences between consecutive high-passed values of the lengths of the acceleration vectors, and divides the sum by the time interval (one second):

$$
A V C=\frac{\sum_{i=1}^{n} \mid \text { length }_{i}-\text { length }_{i-1} \mid}{T_{n}-T_{0}}
$$

$T_{0}$ is the time stamp for the first data sample in the window, and $T_{\mathrm{n}}$ is the time stamp of the last data sample. By applying a threshold to the AVC value, the movement of the appropriate sensor is detected.

\subsection{Activity Recognition}

Seven basic (atomic) activities that can also be interpreted as body postures were studied: standing, sitting, lying, sitting on the ground, on all fours, going down and standing up. We decided only for these activities because they are the most common, atomic, everyday-life activities and are also the most relevant for the detection of falls and distinguishing them from non-falls. Note that sitting and sitting on the ground are two different activities/postures and are not related to the location (e.g. chair, bed, ground) but only to the user's body orientation. 
To recognize the activities of the user, machine learning (ML) was used. The idea of the ML approach was to learn a classification model that will be able to classify the target activities of the person wearing the sensors. The first step in the ML-based AR is the feature extraction procedure. The activities needed to be represented by general features, so that the ML method will also be general and work well on situations different from those in our scenario. Therefore, using the sliding-window technique (described in Subsection 5.1), the data from both types of sensors was first transformed into a number of features. Then, the feature vector was fed into the classification model, which recognized the activity of the user. The ML analysis was performed using the API of the software toolkit WEKA [21]. Among several methods tested, Random Forest yielded the best results in preliminary tests [4, 7]. Random Forest (RF) is an ensemble of decision trees in which the final decision is formed by a majority vote of the tree models [1].

\section{Inertial Features}

This subsection briefly describes the features extracted from the inertial sensors' data and used in the AR [5]. The total number of extracted features per sensor is 25 , i.e., 8 for the gyroscope data and 17 for the accelerometer data, divided into four groups:

- Statistical features (total 20). The Mean Value and the Standard Deviation are extracted for both the acceleration and gyroscope data; additionally, the Root Mean Square (RMS) is calculated only for the accelerometer data. A feature-selection analysis showed that the RMS is a redundant feature for the gyroscope data.

- Movement feature (AVC feature, explained in the Body Movement subsection).

- Sensor inclination angles (total 3). They represent the orientation of the sensor, calculated as the angles between the actual acceleration and each of the axes.

- Difference between the maximum and minimum value of the high-passed acceleration vector in the current data window.

\section{Location Features}

The number of features extracted for the location data does not increase linearly with the number of sensors. The reason for this is that there are features that are extracted for pairs of sensors. Most of the features omit the $\mathrm{x}$ and $\mathrm{y}$ coordinates of the sensors because they refer to the specific location of the user in the room. Our goal was to build a general AR classification model that would not depend on the room's characteristics. The following features were extracted:

- the $z$ (height) coordinate of the sensor,

- the Euclidian distances between each pair of sensors,

- the $z$-distances between each pair of sensors (difference in heights),

- the Euclidian distances between each pair of sensors in the $x y$ plane,

- two velocity-based features: the first one is the absolute velocity of the sensor, and the second one is computed as the velocity of the sensor in the $z$ direction. 


\subsection{Location}

The location system outputs the 3D coordinates of the sensors that are attached to the user's body. In this way it captures properties such as the location of the user in the apartment, e.g., whether the user is on the floor or on the bed, the height of the sensors, etc.

\section{$7 \quad$ Context-based Reasoning}

In this section the context-based reasoning in CoFDILS is presented (Fig. 3). The general idea is that each of the previously described components uses the information from the other two as context, and reasons about the user's situation. Therefore, there are three possible cases: (i) the body acceleration component uses the activity and location as context; (ii) the activity uses the body acceleration and location as context; and (iii) the location uses the activity and body acceleration as context. Additionally, the time component is included, giving another dimension to the reasoning. The time is important for synchronizing the values provided by each component and when analyzing the activities and locations in the time intervals.

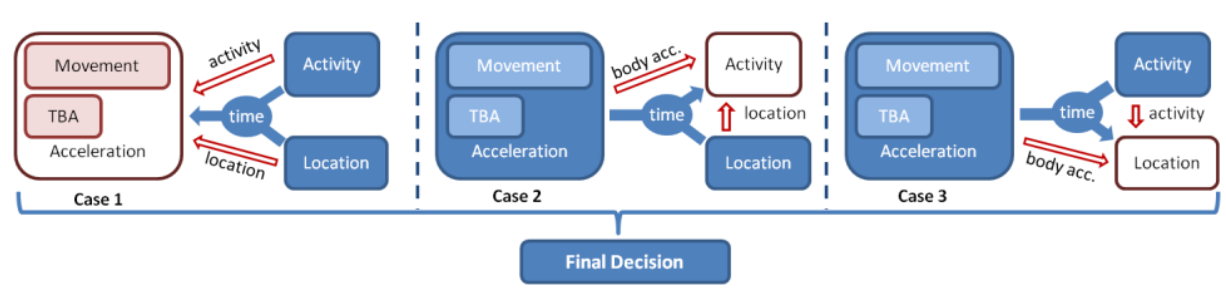

Fig. 3. Context-based reasoning schema.

To explain the basic principle of the context-based reasoning, let us consider the following example in which a user is lying down quickly on a bed, i.e., a non-fall situation. In this case, the acceleration component, i.e., the TBA, recognizes a large acceleration (Case 1 in Fig. 3). If this component reasons by itself, a wrong decision would be formed: a fall would be detected. If the activity of the user is additionally evaluated, the decision would still be wrong (a large acceleration and lying activity = typical fast fall). However, when the location of the user is evaluated (which is the bed), the final decision is corrected into non-fall (quickly lying on the bed).

Besides the example described in the previous paragraph, there are 168 combinations of context dependencies, based on the values of each context component. For a final conclusion, all the potential rules are tested and confirmed. Some rules are primary in the sense that their conclusion is imperative, e.g., normal walking after a large acceleration cancels all the potential fall alarms. Other rules are evaluated using levels of importance, i.e., urgent alarms, normal alarms and warnings, and the most important rule prevails. However, in this study only falls were tested and therefore all alarms had the same level of importance, i.e., urgent. Additionally, in our tests it turned out that only a couple of context-based relations capture most of the context 
information in the FD domain. In particular, to describe a fall we used the activity as a basic piece of information and both location and body acceleration as additional context information. In the next subsections the rules that contain the context dependencies for each sensor type and their combination are presented.

\subsection{Inertial + Location FD}

When the two types of sensors are combined the FD primarily relies on the recognized activity; the additional context information consists of the location and the body movement. As an example, a fall situation is defined by each of the following rules:

- $(A[t 1, t 2]=$ "lying" $) \leftarrow\left(B \_M[t 1, t 2]=\right.$ "no" $\wedge L[t 1, t 2]=$ "floor" $)$;

- $(A[t 1, t 2]=$ "sitting_on_the_ground" $) \leftarrow\left(B \_M[t 1, t 2]=\right.$ "no" $\wedge L[t 1, t 2]=$ "floor" $)$;

- $(A[t 1, t 2]=$ " on_all_fours " $) \leftarrow\left(B \_M[t 1, t 2]=\right.$ "no" $\wedge L[t 1, t 2]=$ "floor" $)$.

where $A[t 1, t 2]$ represents the recognized activity in the time interval $t=[t 1, t 2]$, $B \_M[t 1, t 2]$ represents the body movement in the interval and $L[t 1, t 2]$ is the location in the same interval.

We used assumptions that the elderly do not usually lie or sit on the ground and are not on all fours for more than $t$ seconds while not moving. The value for $t$ was chosen to be 10 seconds. This is long enough for a reliable recognition, but still negligible compared to the time needed for help to arrive.

\subsection{Inertial FD}

In this section we present the context-based reasoning when a conclusion is inferred based on inertial sensors only and therefore a fall situation is defined by the activity and the body accelerations.

Previous experiments showed that it is possible to detect a straightforward (fast) fall by using only TBA; however, lots of false positives appeared in other fall-like events: quickly lying down on a bed, quickly sitting on a chair, etc. Therefore, a potential fall detected by TBA was confirmed by the body movement and additional context information, i.e., the user's activity. As an example, a fall situation is defined by each of the following rules:

- $($ TBA $[t 1]=$ "yes" $) \leftarrow\left(A[t 1, t 2]=\right.$ "lying" $\wedge B \_M[t 1, t 2]=$ "no" $)$;

- $\left(A[t 1, t 2]=\right.$ "sitting_on_the_ground" $\leftarrow\left(B \_M[t 1, t 2]=\right.$ "no" $)$;

- $(A[t 1, t 2]=$ "on_all_fours " $) \leftarrow\left(B \_M[t 1, t 2]=\right.$ "no" $)$.

$\operatorname{TBA}[t 1]=$ "yes", represents the time when a large-acceleration fall pattern is detected.

\subsection{Location FD}

Since the location sensors are better at AR than detecting fall accelerations, FD is based on the activity that may result from a fall, and uses location as the context. The first advantage compared to the stand-alone, inertial FD was the location information: 
the system was aware of some predefined "safe" locations, like the bed. The second advantage was the $z$ coordinate of the sensor location, enabling us to figure out the height of the body and distinguish, for example, sitting on the floor from sitting on a chair. An example of a rule structure is presented here:

- $(A[t 1, t 2]=\{$ "lying" $\vee$ "sitting_on_the_ground" $\vee$ "on_all_fours" $\}) \leftarrow L[t 1, t 2]=$ "floor".

\section{Experimental Setup}

\subsection{Experimental Scenario}

We designed a complex, 15-minute test scenario specifically to investigate events that might be difficult to recognize as falls or non-falls. This scenario (Table 1) was created in consultation with a medical expert. The numbers in parentheses represent the event numbers for easier referencing throughout the text. The events were recorded in a single recording, including all the events.

Table 1. Events in the scenario and their description.

\begin{tabular}{|c|c|c|c|}
\hline & \# & Event & Description \\
\hline \multirow{4}{*}{ 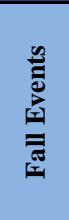 } & (1) & Fast fall (tripping) & Performed in different ways: forwards, backwards or on the sides. \\
\hline & (2) & $\begin{array}{l}\text { Slow fall } \\
\text { (fainting) }\end{array}$ & $\begin{array}{l}\text { Losing consciousness and slowly falling to the ground (trying to } \\
\text { hold onto furniture). }\end{array}$ \\
\hline & (3) & $\begin{array}{l}\text { Falling when trying } \\
\text { to stand up }\end{array}$ & $\begin{array}{l}\text { Trying to stand up from a chair, but has difficulties and slowly } \\
\text { falls to the ground, ending up in a sitting position on the ground. }\end{array}$ \\
\hline & (4) & Sliding from a chair & Person is sliding from a chair and ends up sitting on the ground. \\
\hline \multirow{3}{*}{ 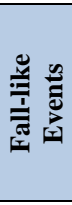 } & (5) & $\begin{array}{l}\text { Quickly lying down } \\
\text { on a bed }\end{array}$ & Person is quickly lying down on a bed. \\
\hline & (6) & $\begin{array}{l}\text { Quickly sitting down } \\
\text { on a chair }\end{array}$ & Person is quickly sitting down on a chair. \\
\hline & (7) & $\begin{array}{l}\text { Searching for some- } \\
\text { thing on the ground }\end{array}$ & $\begin{array}{l}\text { Person first goes on all fours and after this goes to lying on the } \\
\text { ground. }\end{array}$ \\
\hline \multirow{3}{*}{ 焉 } & (8) & Sitting down & Sitting down on a chair normally. \\
\hline & (9) & Lying down & Lying down on a bed normally. \\
\hline & (10) & Walking & Walking sequences between events. \\
\hline
\end{tabular}

Because typical fast falls are easy to detect, only one such fall (1) was included. Three atypical falls (2, 3 and 4) were included to test the use of the contextual activity information, i.e., that a person is not expected to sit/lie on the ground (as opposed to the chair/bed). Furthermore, two events ( 5 and 6 ) we included that involve high acceleration and could thus be misclassified as falls by acceleration-based methods (such as TBA). However, the methods that use the activity and location as contextual information should be able to detect that these are non-fall events. An event (7) was included that involves voluntarily lying on the ground, which could mislead the methods that use information other than acceleration. The events 8, 9 and 10 are normal 
and were included to verify that all the methods work correctly during normal activities.

The experimental scenario was recorded with all 6 inertial and 4 location sensors. Afterwards, the FD was tested with all 1023 combinations of sensors (single type, as well as both types). The scenario was recorded by 11 young, healthy volunteers ( 7 males and 4 females). It was repeated 5 times by each person, resulting in 55 recordings and a total of 550 events for the FD. Testing elderly people was not feasible because of the scenario complexity and for safety reasons, but the volunteers were advised how to act by the medical expert.

Additionally, the data for three more people was recorded for tuning the basic parameters, e.g., thresholds, preliminary tests, choosing the best algorithms (details can be obtained from the authors).

\subsection{Evaluation Technique}

To evaluate the FD, one must decide how to weight the missed falls and the false alarms. Both are important: missing a fall may endanger a person's health, while false alarms make the system unlikely to be used in real life. Therefore, we used the Fmeasure $(\mathrm{F})$, which weights missed falls and false alarms equally. It is defined as a harmonic mean of recall (the percentage of the events recognized as falls/non-falls from all falls/non-falls events) and precision (the percentage of the events truly being falls/non-falls of all predictions for falls/non-falls). However, for more detailed results, the true positive and true negative rates are presented for the fall and non-fall events, respectively.

\section{$9 \quad$ Experimental Results and Discussion}

Fig. 4 presents a matrix $(5 \times 7)$ representation of the best sensor combinations. The inertial sensors are shown on the $\mathrm{x}$ axis and the location on the $\mathrm{y}$ axis. Each rectangle in the matrix contains the sensor placements and the achieved F-measure as a percentage (marked with F in Fig. 4). For example, the $(2 \times 3)$ rectangle represents a combination of 2 location and 3 inertial sensors. It is the best of all combinations according to the F-measure $=99.7 \%$. The dotted lines (diagonal) connect the rectangles that have the same number of sensors. Along each dotted line the best (according to the Fmeasure) rectangle is marked with a white circle. These rectangles represent the best combination given the number of sensors.

Tests of the statistical significance were performed. The best sensor combinations for each number of sensors (white circles in Fig. 4) and each sensor type (rectangles on the axes) were tested separately. Because of the small number of folds (11) and because the individual samples (folds) are paired (the same person's data for each combination), we used the paired Student's T-test with a significance level of 5\%.

Analyzing the results achieved with the inertial sensors only (horizontal axis rectangles), one can see that the only important improvement is detected when using two sensors instead of one. After this, adding up to five sensors did not significantly im- 
prove the F-measure; including a sixth sensor even decreased the performance. In another observation, the chest sensor is the most suitable for inertial FD, because it is in all the sensor combinations.

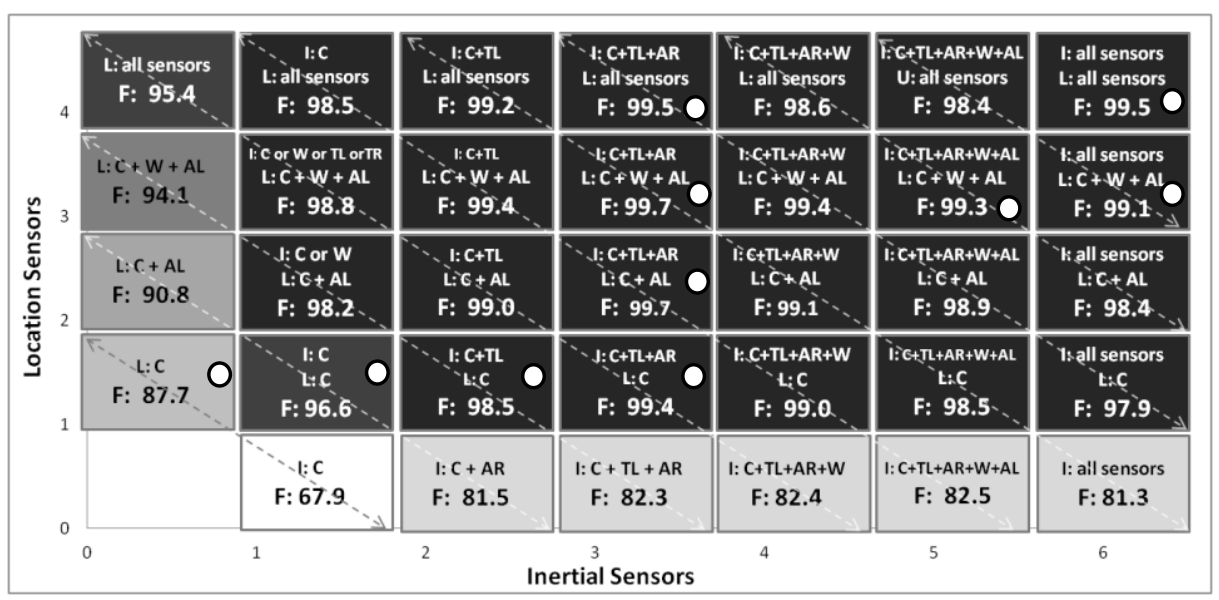

Fig. 4. Matrix representation of the best sensor combinations using the Inertial (I) and Location (L) sensors. F - overall F-measure, C - Chest, W - Waist, RA - Right Ankle , LA - Left Ankle , RT - Right Thigh, LT - Left Thigh.

For the location FD, an increase in the number of sensors increases the performance all the way. The statistical tests proved that there is a significant difference in the performance of a system using one, two, three and four location sensors. Like with the inertial FD, the chest is the best-performing placement.

The statistical tests for the combined FD showed that the difference in performance is statistically significant only when the system is using two and three sensors. Four sensors or more do not significantly increase the performance of the system.

The parts of the graph with a smaller number of sensors are of the greatest interest for practical use. The combination of sensors clearly outperforms the individual sensor types. For example, the performance values of the system using two sensors are $81.5 \%$ and $90.8 \%$, for the inertial and location sensors, respectively. Their combination improves these results by 15 p.p. and 6 p.p., respectively. This is the case for each number of sensors (dotted lines): the combination of two sensor types is better than each of the types used separately. Furthermore, one can put the two sensor types in one sensor enclosure. The performance of the system using only one sensor type is $68 \%$ and $88 \%$ for the inertial and location sensor, respectively. Combining them in one enclosure improves these results by 29 p.p. and 9 p.p., respectively.

The rest of the discussion is a detailed analysis of the results achieved by the simplest and the best (statistically significant) combinations of the inertial-only, locationonly and both types of sensors. The sensor types and placements are shown in Table 2 and the results are presented in Table 3. 
Table 2. The simplest and the best (statistically significant) combinations of the inertial-only, location-only and both types of sensors.

\begin{tabular}{|l|l|l|}
\hline Sensor types/placements & The simplest combination & The best combination \\
\hline Inertial sensors & Chest & Chest + Right ankle \\
\hline Location sensors & Chest & All four sensors \\
\hline Combined sensors & $\begin{array}{l}\text { Inertial: Chest } \\
\text { Location: Chest }\end{array}$ & $\begin{array}{l}\text { Inertial: Chest + Right ankle } \\
\text { Location: Chest }\end{array}$ \\
\hline
\end{tabular}

The events in Table 3 are divided into two groups: fall and non-fall (fall-like and normal) events. The number for each event is the percentage of all fall/non-fall events being correctly recognized as fall/non-fall (true-positive rate/true-negative rate). The last row represents the overall F-measure.

Table 3. Detailed FD results for each event and each context-based FD method.

\begin{tabular}{|c|c|c|c|c|c|c|c|}
\hline & \multicolumn{6}{|c|}{ Context-based Reasoning } \\
\hline & & \multicolumn{2}{|c|}{$\begin{array}{c}\text { Inertial } \\
\text { (Activity + TBA + } \\
\text { Movement) }\end{array}$} & \multicolumn{2}{|c|}{$\begin{array}{c}\text { Location } \\
\text { (Activity + Location) }\end{array}$} & \multicolumn{2}{|c|}{$\begin{array}{c}\text { Combination } \\
\text { (Activity + TBA + } \\
\text { Movement + Location) }\end{array}$} \\
\hline & & \begin{tabular}{|l|} 
simplest \\
\end{tabular} & best & simplest & best & simplest & best \\
\hline \multirow{4}{*}{ 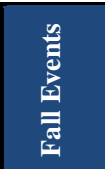 } & (1) Tripping - Quick falling & 100 & 100 & 96 & 100 & 100 & 100 \\
\hline & (2) Fainting - Falling slowly & 11 & 11 & 100 & 100 & 100 & 100 \\
\hline & (3) Falling from a chair slowly & 68 & 98 & 95 & 95 & 99 & 99 \\
\hline & (4) Sliding from a chair & 72 & 99 & 97 & 97 & 98 & 99 \\
\hline \multirow{3}{*}{ 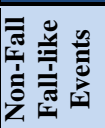 } & (5) Sit down quickly on a chair & 55 & 97 & 75 & 89 & 91 & 98 \\
\hline & (6) Searching on the ground & 85 & 88 & 25 & 78 & 80 & 89 \\
\hline & (7) Quickly lying down on a bed & 34 & 34 & 100 & 100 & 100 & 100 \\
\hline \multirow{4}{*}{ 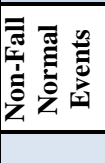 } & (8) Sitting normally & 68 & 98 & 80 & 93 & 93 & 98 \\
\hline & (9) Lying normally & 100 & 100 & 100 & 100 & 100 & 100 \\
\hline & (10) Walking & 97 & 100 & 92 & 97 & 100 & 100 \\
\hline & Overall F-measure in \% & 67.9 & 81.5 & 87.7 & 95.4 & 96.6 & 98.5 \\
\hline
\end{tabular}

The first two columns show the results achieved by the inertial FD. The first event in Table 3, tripping, is a typical fall that was recognized accurately because of the TBA rule. The second event, which is falling slowly, was difficult to recognize because of the low acceleration during this event. For this event, additional contextual information was necessary (e.g., the location of the user). The effect of the activity information of the user can be seen in the fall events that end with sitting on the ground (events 3 and 4). In these cases the AR model correctly recognized sitting on the ground. On the other hand, this has a negative impact on the performance when the sitting event is analyzed (events 5 and 8). In this case, the AR model is not accurate enough and recognizes sitting on the ground, resulting in a false positive. This issue is solved by including more sensors, which improves the AR method (e.g., the column Inertial-best).

The location FD was using the activity and the location information. But because of the location, it recognized all falls with high accuracy (events 1 to 4). However, 
some problems still exist in non-fall events, because of the relatively low accuracy of the AR model. Namely, sitting (events 5 and 8 ) and searching on the ground (event 6) were misclassified as sitting on the ground or lying (on the ground), causing the system to detect a fall during non-fall events. Improvements in the performance can be seen when the number of sensors is increased (the column Location-best), due to the improvements in the AR method.

The last two columns show the results achieved with the combination of both types of sensors and the full context as presented in Subsection 7.1. The improvements are clear in all of the events. The overall performance when two sensors (one inertial and one location) are used is $96.6 \%$. Some problems only appear in non-fall events that end with sitting (5 and 8) and the searching on the ground event (6). The reason lies in the AR method, which misrecognizes the appropriate activities (sitting and on all fours). These problems are solved by including one more inertial sensor, which significantly improves the AR model and consequently the FD (the last column in Table 3).

To summarize, the best-performing combination of sensors is two inertial sensors (chest + left thigh) and one location sensor (chest). With this combination, the context-based FD method achieves an F-measure of $98.5 \%$, mostly because of the location sensor, which provides the context location information. However, the best practical solution with the minimum number of sensors is the combination of the chest inertial and chest location sensor, achieving an F-measure of $96.6 \%$.

Finally, two commonly used methods in the literature, the threshold-based approach (TBA) and the machine-learning approach (MLA), are tested for comparison. The results are shown in Fig. 5, by presenting the true-positive or true-negative rate for each fall or non-fall event, respectively.

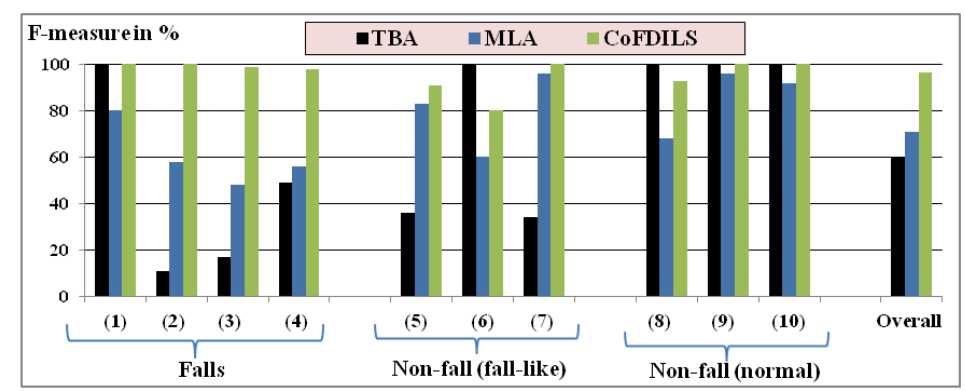

Fig. 5. Comparison of the FD results achieved by: our Context-based approach, the Machine-learning approach (MLA), and Threshold-based approach (TBA). The event numbers correspond to the events given in Table $\mathbf{1}$.

The TBA is described in Subsection 6.2. More details about the MLA can be found in our previous work (Luštrek et al. [14]). The basic principle of MLA is that a machine-learning model is trained to detect a fall event. In our case, features extracted from the chest-inertial and chest-location sensor data were used. Therefore, the contextual location information was implicitly (through features) introduced in the MLA.

The overall results showed that our method, in which the context is explicitly encoded with rules, outperformed the other two methods, which use: implicit context 
information (MLA) or only accelerations (TBA). The TBA outperformed our CoFDILS only in two events $(6,8)$; however, this was due to the one-sided performance of the TBA (detects only large accelerations) at the expense of the overall performance.

\section{Conclusion}

We presented a novel approach, i.e., CoFDILS, to fall detection by combining inertial and location sensors using a general context-based schema. The method exploits three components, i.e., the activity of the user, the body accelerations and the location, to detect a fall situation. The decision of each component based on the same type of input data is re-evaluated in the context of the other two components. There are 168 combinations of context dependencies, based on the values for each component; however, in our tests it turned out that only a couple of context-based relations capture most of the context information in the FD domain. Currently, the context-based reasoning rules were provided by an expert. The automation of learning the context relation is considered as future work.

We tested the performance with all possible combinations of the six inertial and four location sensors to find the best sensor placements, using the context-based decision schema. The evaluation was performed on a complex test scenario; it included real life, realistic events that were difficult to recognize as falls or non-falls. The results showed that by combining the two types of sensors it is possible to detect complex fall situations by using the activity and the context information from both types of sensors. It is essential that both sensor types are employed, since they provide complementary information about the user's situation. Finally, the best practical solution is the chest-sensor placement with a single sensor enclosure, combining one inertial and one location sensor.

Acknowledgements. This work was partly supported by the Slovenian Research Agency under the Research Programme P2-0209 Artificial Intelligence and Intelligent Systems and partly from the European Community's Framework Programme FP7/2007-2013 under grant agreement No.214986. The authors would like to thank Boštjan Kaluža for the helpful suggestions and discussions.

\section{References}

1. Breiman, L. Random forests. Machine Learning, 45, (2001), 5-32.

2. Dey, A., Salber, D., Abowd, G., Futakawa, M. The conference assistant: Combining context-awareness with wearable computing. In International Symposium on Wearable Computers, San Francisco, USA, (1999).

3. Friedman, S. M., Munoz, B., West, S. K.; Rubin, G. S., Fried, L. P. Falls and Fear of Falling: Which Comes First? A Longitudinal Prediction Model Suggests Strategies for Primary and Secondary Prevention, Journal of the American Geriatrics Society, (2002), 1329-1335. 
4. Gjoreski, H., Gams, M., Chorbev, I. 3-axial accelerometers activity recognition. ICT Innovations, (2010), 51-58,.

5. Gjoreski, H., Luštrek, M., Gams, M. Accelerometer Placement for Posture Recognition and Fall Detection. The 7th International Conference on Intelligent Environments, (2011).

6. Gillespie, L. D., Robertson, M. C., Gillespie, W. J., Lamb, S. E., Gates, S.; Cumming, R. G., Rowe, B. H. Interventions for preventing falls in older people living in the community (Review) The Cochrane Library, 4 (2009).

7. Gimon, D., Gjoreski, H., Kaluža, B., Gams, M. Using accelerometers to improve positionbased activity recognition. $13^{\text {th }}$ International Multi-Conference Information Society, (2010), 15-18.

8. Jantaraprim, P., Phukpattaranont, P., Limsakul, C., Wongkittisuksa, B. Evaluation of fall detection for the elderly on a variety of subject groups. The $3 \mathrm{rd}$ International Convention on Rehabilitation Engineering \& Assistive Technology, (2009).

9. Kaluza, B., Dovgan, E. Glajenje trajektorij gibanja cloveskega telesa zajetih z radijsko tehnologijo. Information Society, Ljubljana, Slovenia (2009).

10. Khawandi, S., Daya, B., Chauvet, P. Automated monitoring system for fall detection in the elderly, International Journal of Image Processing (IJIP), 4(5) (2010).

11. Li, Q., Stankovic, J., Hanson, M., Barth, A. T., Lach, J., Zhou, G. Accurate, Fast Fall Detection Using Gyroscopes and Accelerometer-Derived Posture Information. Sixth International Workshop on Wearable and Implantable Body Sensor Networks, (2009), 138-143.

12. Li, Q., Stankovic, J. Grammar-based, posture- and context-cognitive detection for falls with different activity levels. WH '11: 2nd Conference on Wireless Health, (2011).

13. Lin, H.-T., Hsieh, Y.-J., Chen, M. C., Chang, W. ActionView: a movement-analysis ambulatory monitor in elderly homecare systems. IEEE International Symposium on Circuits and Systems, (2009), 3098-3101.

14. Luštrek, M., Gjoreski, H., Kozina, S., Cvetković, B., Mirchevska, V., Gams, M. Detecting Falls with Location Sensors and Accelerometers, Twenty-Third IAAI Conference, (2011).

15. Machajdik, J., Zambanini, S., Kampel, M. Fusion of Data from Multiple Cameras for Fall Detection. Workshop on Behaviour Monitoring and Interpretation, BMI'10, 1-7 (2010).

16. Rubenstein, L. Z., Josephson, K. R. The epidemiology of falls and syncope. Clinics in Geriatric Medicine, (2002), 18(2):141-158.

17. Shan, S., Yuan, T. A. Wearable Pre-impact Fall Detector using Feature Selection and Support Vector Machine. Systems, (2010), 1686-1689.

18. Tinetti, M. E., and Williams, C. S. Falls, Injuries Due to Falls, and the Risk of Admission to a Nursing Home. The New England Journal of Medicine (1997) 337: 1279-1284.

19. Ubisense RTLS website. http://www.ubisense.net (accessed June 2012).

20. World Health Organization (WHO). Global brief for World Health Day 2012, Good health adds life to years. http://whqlibdoc.who.int/hq/2012/WHO_DCO_WHD_2012.2_eng.pdf (accessed June 2012).

21. Witten, I., Frank, E. Data Mining: Practical machine learning tools and techniques. Morgan Kaufmann. 2nd Edition, (2005).

22. XSens sensors website. http://www.xsens.com (accessed June 2012).

23. Zinnen, A., Wojek, C., Schiele, B. Multi Activity Recognition Based on BodymodelDerived Primitives, 4th Int. Symposium on Location and Context Awareness, (2009).

24. Zhang, T., Wang, J., Xu, L., Liu, P. Fall Detection by Wearable Sensor and One-Class SVM. Intelligent Computing in Signal Processing and Pattern Recognition, 345, (2006), 858-863. 